\title{
A painful knee in an immunocompromised patient
}

Department of Orthopaedics, Eastbourne District General Hospital, Eastbourne, UK RM Pattison

The Department of Plastic and

Reconstructive

Surgery, St George's

Hospital, London

SW17 0QT, UK

MA O'Donnell

ALH Moss

Department of Haematology, Royal Marsden Hospital, Sutton, Surrey, UK R Powles

Correspondence to $\mathrm{Mr}$ RM Pattison, 17 Thames Haven, Portsmouth Road, Surbiton, Surrey KT6 4JA, UK

Accepted 13 November 1995

\section{RM Pattison, MA O’Donnell, R Powles, ALH Moss}

A 34-year old Turkish man underwent an allogeneic bone marrow transplant as treatment of his chronic myeloid leukaemia. Five weeks later he was re-admitted complaining of severe pain in his previously normal left knee. On examination he was pyrexial, the knee was warm and slightly swollen with a small effusion but had a normal range of movement. Initial X-rays of the knee showed soft tissue swelling only but in subsequent films there was an increasing amount of air in the joint and surrounding soft tissues with scalloping of the femoral condyles. Joint aspiration produced only $5 \mathrm{ml}$ of sterile, blood-stained fluid.

Over the next two weeks the knee became more swollen with dusky discolouration anteriorly, that developed into a large black, necrotic area (figure 1).

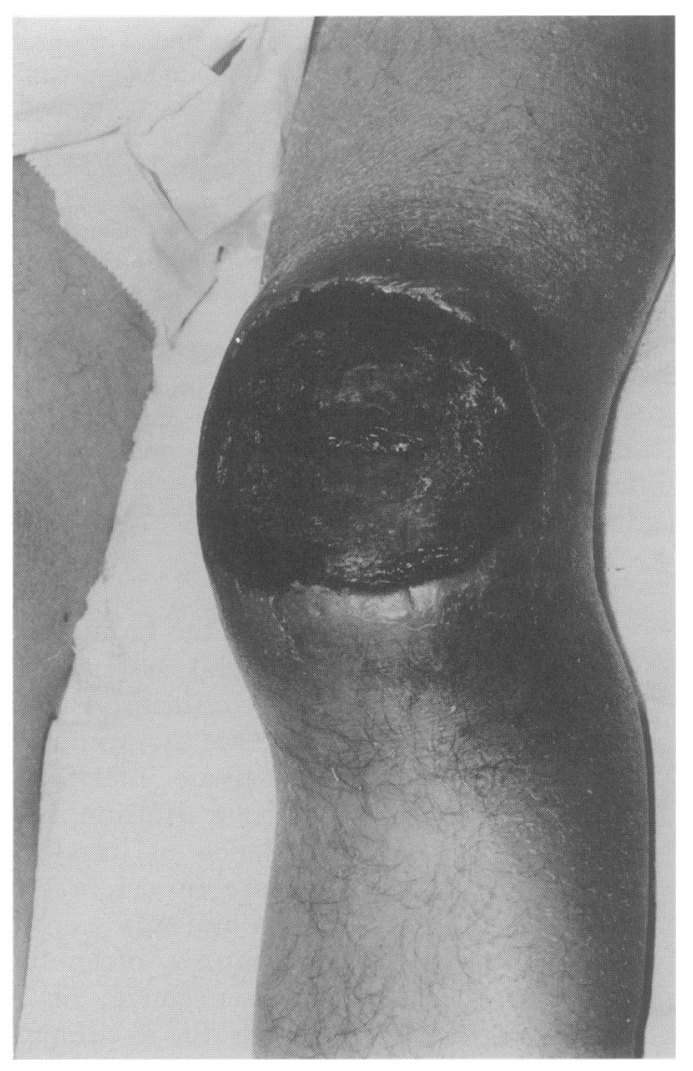

Figure 1 Left knee of patient

\section{Questions} 2 How should this condition be treated?
1 What diagnostic tests should be performed? 


\section{Answers}

\section{QUESTION 1}

The blood count showed a persistent neutropenia but was otherwise unremarkable. The initial tissue biopsy was negative but microbiological swabs taken from the knee grew Aspergillus fumigatus. The diagnosis was of cutaneous infection with $A$ fumigatus. Histological examination of tissue obtained post mortem showed ulcerated, congested skin infiltrated with extensive branching fungal hyphae and plugs in dermal blood vessels, confirming the diagnosis (figure 2).

\section{QUESTION 2}

Antimicrobial therapy was started with $\mathrm{AmBi}$ some $3 \mathrm{mg} / \mathrm{kg}$ daily and miconazole cream topically. A fumigatus infections are not confined by tissue planes and can be dramatically haemorrhagic so early surgical excision should be considered. In this case the patient developed pneumonia, hepatic and renal failure and died three weeks after developing this cutaneous aspergillosis.

\section{Discussion}

A fumigatus is a commonly isolated respiratory pathogen in patients with leukaemia, neutropenia or on immunosuppressive therapy. ${ }^{1}$ In the presence of decreased cellular immunity, the focal respiratory tract infection can become invasive, leading to disseminated disease af-

Figure 2 Skin infiltrated with fungal hyphae and plugs
Microorganisms that form gas in soft tissues

- Clostridium perfringens, septicum and novyi

- $\mathrm{Cl}$, histolyticum, fallax and bifermentans may

have a synergistic role in gas gangrene

- Aspergillus fumigatus

\section{Box 1}

Diagnosis and treatment of cutaneous aspergillosis

- tissue biopsy of the edge of the lesion for histological and microbiological examination

wound swabs less reliable

- high-dose intravenous amphotericin

(AmBisome) and topical antifungals

- prompt surgical excision is mainstay of treatment

\section{Box 2}

The common organisms that cause septic arthritis in immunocompetent patients also affect the immunocompromised. In addition the following organisms have been described:

- Salmonella enteritidis

- Haemophilus influenzae type B

- meningococcus

- Neiserria gonorrhoea

- Mycobacterium tuberculosis

- atypical mycobacteria

- Candida albicans

- coccidioidomycosis

- cryptococcus

- blastomycosis

Box 3

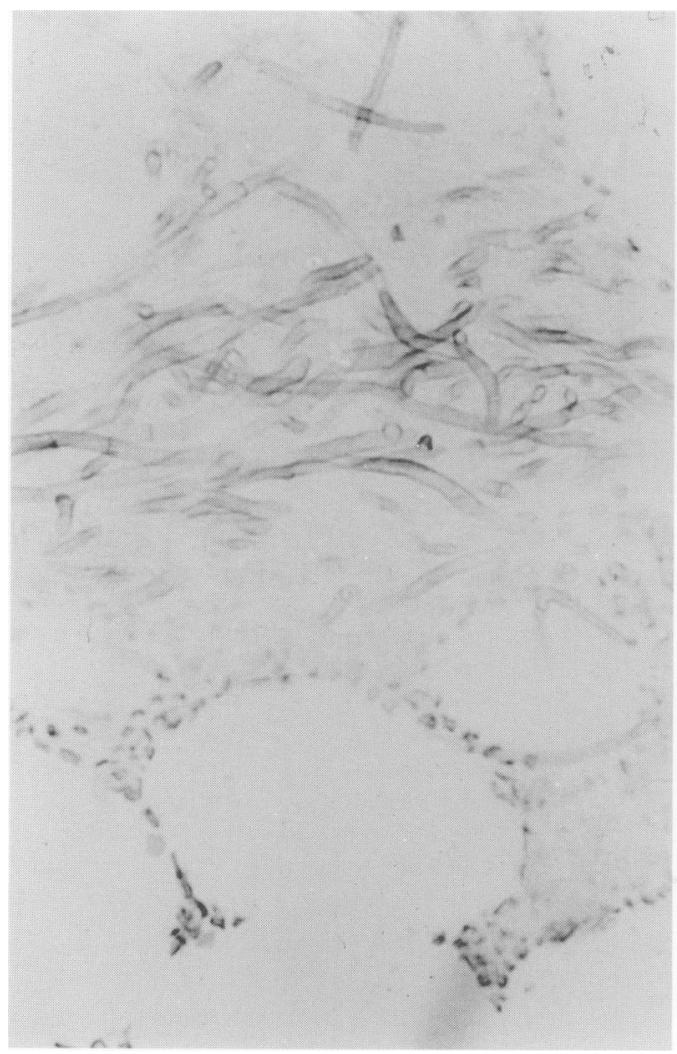

fecting the brain, liver or skin. In the musculoskeletal system it has been implicated in septic arthritis, osteomyelitis and bursitis. ${ }^{2,3}$ In this case the primary pathology was a localised soft tissue infection around the knee with a reactive effusion in the joint. Radiographic evidence of intralesional air is a diagnostic feature in cerebral or pulmonary aspergillomata and was seen in this case (box 1).

Cultures of sputum of tissue samples are often negative, or may produce misleading results due to contamination. An adequate soft tissue biopsy should be taken in atypical cases. In this case histological examination of postmortem tissue was diagnostic (box 2).

Although the differential diagnosis of a hot, swollen joint in this group of patients is already long (box 3), this case demonstrates that an apparent monoarthritis due to infection of the surrounding soft tissues with joint pain and a reactive effusion should be considered.

\section{Final diagnosis}

Cutaneous aspergillosis of the knee

Keywords: aspergillosis

3 Ornvold K, Paepke J. Apergillus terreus as a cause of olecranon bursitis. Eur $\mathcal{f}$ Clin Am Pathol 1992; 97: 114-6.
Bodey GP, Vartavarian S. Aspergillosis. Eur f Clin Microbio Infect Dis 1989; 8: 413-37.

Horsburgh CR, Cannady PB, Kirkpatrick CH. Treatmen of fungal infections in the bones and joints with Ketoconazole. F Infect Dis 1983; 147: 1064-9. 